\title{
Dynamics and Stability of Axially Symmetric Atomic Clouds in Magneto-Optical Trap
}

\author{
Received: 25.12.2020 \& Accepted: 03.03.2021
}

\author{
L.G.F. SOARES* AND F. HAAS
}

Instituto de Física, Universidade Federal do Rio Grande do Sul, Av. Bento Gonçalves 9500, 91501-970 Porto Alegre, RS, Brasil

Doi: 10.12693/APhysPolA.139.659

*e-mail: guti_01@live.com

\begin{abstract}
The three-dimensional nonlinear dynamics of atomic clouds in a magneto-optical trap is analyzed in terms of a self-consistent fluid formulation using a variational approach. A Lagrangian density is proposed in the case where thermal and multiple-scattering effects are both relevant, in addition to damping. For closure, an adiabatic equation of state is assumed. After adopting a Gaussian profile for the fluid density and appropriated spatial dependencies of the scalar potential and potential fluid velocity field, a set of ordinary differential equations is derived. The linear stability analysis and the prominent features of the associated normal modes are characterized. Unlike previous treatments found in the literature, the current analysis applies to non-spherically symmetric trapped atomic gases. In addition, the use of a variational approach for a dissipative system is a further distinctive feature of the work. The paper is restricted to potential flows.
\end{abstract}

topics: trapped atoms, time-dependent variational method, magneto-optical trap, normal modes

\section{Introduction}

The optical confinement of large samples of alkaline atom clouds to produce Bose-Einstein condensates (BECs) is a significant task [1]. Magnetooptical traps (MOTs) are one of the most successful methods for this purpose. The environment that allows the confinement of alkaline atoms originates from the potential well created by a magnetic field gradient (produced by anti-Helmholtz coils) and an intersection of three pairs of orthogonally positioned circularly polarized beams that cool the sample [2,3]. Such confinement involves the combined effects of the magnetic trapping and Doppler cooling mechanisms $[4,5]$. MOTs are also essential in the realization of optical lattices $[6,7]$, observation of collective quantum effects [8], selfinduced electron trapping in freely expanding neutral plasmas [9], coherent excitation of Rydberg states in cold atomic gases [10], atomic clocks and performance enhancement $[11,12]$ and they also provide a medium with several self-organized structures [13].

At the low saturation regime, the dynamics of trapped gases share similarities with confined non-neutral plasmas such as an antiproton gas in a Penning-Malmberg trap cooled to extremely low temperatures [14]. There are also analogies with the classical limit of an electron gas in a semiconductor quantum well in the mean-field approximation [15] and astrophysical models for pulsating stars [5, 16]. Such similarities make it possible to take moments of the distribution function from the kinetic theory, deriving hydrodynamic equations for the macroscopic quantities, which can then be closed assuming a state equation. Therefore, the atomic clouds in MOTs can be formally described using well known hydrodynamical models from plasma physics. In some situations, this can be done when the diffusive term in the Fokker-Planck equation can be neglected, which is valid when the laser light is so intense that the effects of absorption and radiation trapping forces take over the photon exchange with the cooling laser [17-20].

Previous studies rely on radially symmetric configurations, restricted to the linear approximations [17-19], direct numerical analysis [21-23] or specific analytic methods [24]. However, it must be emphasized that the spherical symmetry assumption does not strictly apply to MOTs [25] since anti-Helmholtz coils create an axially symmetric magnetic field, see further (12). This axial symmetry motivates our present analysis of anisotropic trapped gases. For this purpose, the Doppler limit is assumed, i.e, the lower temperature reached is delimited by the Doppler cooling limit. Nevertheless, sub-Doppler temperatures can be obtained through Sisyphus cooling (when considering the polarization 
of the laser beams) or evaporative cooling. The treatment consists of the minimization of the action functional, reducing the problem to a set of coupled ordinary differential equations. In addition, the linear stability analysis of the normal modes will be discussed.

In the hydrodynamic model, the external gradient magnetic field provides the harmonic confinement. This magnetic confinement arises from the Zeeman effect, which splits the atomic energy levels. Such splitting depends on the configuration of the magnetic fields. The most commonly used magnetic trap to confine atoms is the quadrupole field created by anti-Helmholtz coils [2, 26-30]. However, this kind of trap leads to a loss of particles since the magnetic field at the center of the trap is zero. The escape mechanism is known as the spin-flip Majorana transition, which can be a problem when creating large samples of BECs. To overcome this issue, it is possible to modify MOTs by adding a time-dependent rotating bias field in the $x y$-plane (TOP trap) [31-33] or using a pair of Helmholtz coils with a series of wires around the two coils [34, 35] (the Ioffe-Pritchard trap). All of these traps share azimuthal symmetry [36], which will be adopted here as in [22]. Additionally, the presence of the lasers provides a damping mechanism, which is also included in our treatment. The repulsive collective force and the pressure term tend to expand the atomic cloud. Experiments with MOTs are usually performed in the temperaturelimited (TL) regime or in the multiple-scattering (MS) regime, which dominates the long range interactions. These regimes depend on the number of confined atoms. For certain conditions, both effects can be important and in typical experiments with an intense magnetic gradient field the underdamped regime can be accessed [24].

The dynamical study of nonlinear systems can be simplified using variational methods, as in BoseEinstein condensates [37-43] and quantum electron gases $[15,44,45]$. In this context, the timedependent variational method allows us to assess nonlinear and time-dependent dynamics by adopting a trial function, frequently a Gaussian Ansatz.

Since the Doppler cooling produces a damping force, the proposed Lagrangian density has a time-dependent exponential factor, and is linearly dependent on the velocity potential. This is similar to the Caldirola-Kanai non-conservative variational approach for the damped harmonic oscillator [46, 47].

The present article is organized as follows: the basic set of hydrodynamic equations and the Lagrangian density is introduced in Sect. 2. There, a suitable time-dependent Ansatz is proposed for the number density, which reduces the problem to a set of coupled nonlinear second-order equations for the dynamical parameters of the Gaussian atomic cloud. Section 3 applies the results to MOTs and introduces a pseudo-potential function, which allows an accurate linear stability analysis, and the assessment of the normal modes, to be performed in Sect. 4. The conclusions are drawn in Sect. 5.

\section{Basic model and time-dependent variational method}

A trapped gas in a MOT can be described by the following hydrodynamic equations,

$$
\begin{aligned}
& \frac{\partial n}{\partial t}+\nabla \cdot(n \boldsymbol{v})=0 \\
& \frac{\partial \boldsymbol{v}}{\partial t}+(\boldsymbol{v} \cdot \nabla) \boldsymbol{v}= \\
& \quad-\nu \boldsymbol{v}-\frac{1}{m} \frac{\nabla p}{n}-\frac{1}{m} \nabla V_{h}+\frac{1}{m} \nabla V_{c}
\end{aligned}
$$

$\nabla^{2} V_{c}=Q n$,

where $V_{h}=m\left(\omega_{x}^{2} x^{2}+\omega_{y}^{2} y^{2}+\omega_{z}^{2} z^{2}\right) / 2$ is the external confining potential. The respective (1), (2), and (3) are the continuity, momentum and Poisson equations. The system is composed of atoms (atomic mass $m$ ) with a number density $n=n(\boldsymbol{r}, t)$, a fluid velocity field $\boldsymbol{v}=\boldsymbol{v}(\boldsymbol{r}, t)$ and the pressure $p$.

When the ratio between the intensities of the incident laser or saturation parameter $s_{\text {inc }} \ll 1$, the MOT force can be described as a harmonic force (with an angular frequency which is not the same in all directions), and a dissipative force with the damping coefficient $\nu$. These forces originate, respectively, from the Zeeman shift and Doppler cooling. In this context, the angular frequency and the damping coefficient can be written in terms of the atomic transitions and confinement parameters,

$$
\begin{aligned}
& \omega_{z}=\sqrt{2} \omega_{\perp}=\sqrt{\frac{2 \nu \mu}{k_{L}}}, \\
& \nu=-\frac{8 \hbar k_{L}^{2} s_{\mathrm{inc}} \Delta}{m \Gamma\left(1+4 \Delta^{2} / \Gamma^{2}\right)^{2}},
\end{aligned}
$$

where $k_{L}$ is the amplitude of the laser wave vector, $\hbar$ is the reduced Planck constant, $\Delta$ is the detuning frequency between the laser frequency and the atomic transition frequency, and $\Gamma$ is the natural line width of the transition used in the cooling process. Also, $\mu=\mu_{\mathrm{B}} B_{0} / \hbar$, with $\mu_{\mathrm{B}}$ being the Bohr magneton and $B_{0}-$ the intensity of the gradient field. The numerical factor $\sqrt{2}$ comes from the configuration of the magnetic field created by a pair of anti-Helmholtz coils, namely $\boldsymbol{B}=B_{0}\left(x \hat{e}_{x}+y \hat{e}_{y}-2 z \hat{e}_{z}\right)$, so that $\omega_{x}=\omega_{y}=\omega_{\perp}$. For the other forms of magnetic traps, it is necessary to derive appropriate expressions of the confinement force. In MOTs, the red detuning $(\Delta<0)$ provides $\nu>0$.

The self-consistent potential $V_{c}$ is associated with the collective force, satisfying a Poisson equation with an effective charge of the atoms given by $q=\sqrt{\epsilon_{0} Q}=\sqrt{\left(\sigma_{R}-\sigma_{L}\right) \sigma_{L} I_{0} / c}$, where $\epsilon_{0}$ is the vacuum permittivity, $c$ is the speed of light and $I_{0}$ is the total intensity of the six laser beams, while $\sigma_{\perp}$ 
and $\sigma_{R}$ represent the emission and absorption cross sections, respectively [48]. This potential is a contribution of two parts, the first being an attractive potential created by the imbalance of the absorption of light when the backward and forward laser intensities are locally different. The second part comes from a repulsive potential. This repulsion is a consequence of rescattering photons that tend to push away nearby atoms $[50,51]$. In typical experiments $[3,49]$, the repulsion dominates over the attractive force $(Q>0)$.

For the sake of definiteness, assume an adiabatic equation of state $p=n_{0} k_{\mathrm{B}} T\left(n / n_{0}\right)^{5 / 3}$ for fast processes, where $n_{0}$ is the reference number density (that will be better defined later), $k_{\mathrm{B}} T$ is the reference thermal energy value where $k_{\mathrm{B}}$ is the Boltzmann constant, and the respective exponent corresponds to the adiabatic coefficient $\gamma=(d+2) / d=5 / 3$ for the dimensionality $d=3$.

The problem of solving the set (1)-(3) for an irrotational velocity $(\nabla \times \boldsymbol{v}=0)$ can be reinterpreted as a variational problem corresponding to the minimization of the action functional $S=\int \mathrm{d} t \mathrm{~d}^{3} r \mathcal{L}$, specified by the Lagrangian density

$$
\begin{aligned}
\mathcal{L} & =\mathrm{e}^{\nu t}\left[m n\left(\frac{1}{2}|\nabla \phi|^{2}+\frac{\partial \phi}{\partial t}+\nu \phi\right)\right. \\
& \left.+n\left(V_{h}-V_{c}\right)-\frac{\left(\nabla V_{c}\right)^{2}}{2 Q}+\int \mathrm{d} n \int \frac{\mathrm{d} p}{n}\right],
\end{aligned}
$$

where the independent fields are the velocity potential $\phi=\phi(\boldsymbol{r}, t)$, so that $\boldsymbol{v}=\nabla \phi$, the number density $n=n(\boldsymbol{r}, t)$, and the self-consistent potential $V_{c}=V_{c}(\boldsymbol{r}, t)$. Indeed, it can easily be shown that the minimization of the respective Lagrangian density corresponding to (4) and (5), with respect to the fields $\phi, n$ and $V_{c}$, respectively, yields the continuity, momentum and Poisson equations. The use of the Lagrangian formalism for dissipative systems is not unusual for discrete dynamical systems $[46,47]$, but it is less frequent for continuous systems.

A normalized Gaussian Ansatz is adopted,

$$
n(\boldsymbol{r}, t)=\frac{A}{\alpha_{x} \alpha_{y} \alpha_{z}} \exp \left(-\frac{\rho^{2}}{2}\right),
$$

where $A=N /(2 \pi)^{\frac{3}{2}}, N$ is the number of confined atoms and

$$
\begin{aligned}
& \rho(\boldsymbol{r}, t)=\left[\frac{\left(x-d_{x}(t)\right)^{2}}{\alpha_{x}^{2}(t)}\right. \\
& \left.+\frac{\left(y-d_{y}(t)\right)^{2}}{\alpha_{y}^{2}(t)}+\frac{\left(z-d_{z}(t)\right)^{2}}{\alpha_{z}^{2}(t)}\right]^{1 / 2} .
\end{aligned}
$$

The Gaussian form reflects the atomic confinement and is amenable for an analytic treatment [15, 44]. The time-dependent coordinates $d_{i}(t)$ and $\alpha_{i}(t)$, with $i=x, y, z$, respectively, give the position of the center of mass and the width of the atomic cloud in different directions. In addition, define the reference number density as $n_{0}=N /\left(\alpha_{x 0} \alpha_{y 0} \alpha_{z 0}\right)$, where $\alpha_{i 0}=\alpha_{i}(0)$.
Direct substitution of the Ansatz in the continuity equation (1) leads to an exact solution for the velocity field, given by

$$
v_{i}=\frac{\dot{\alpha}_{i}}{\alpha_{i}}\left(r_{i}-d_{i}\right)+\dot{d}_{i}
$$

where $v_{i}$ is the $i$ component of the fluid velocity field. Since $\boldsymbol{u}=\nabla \phi$, the scalar field $\phi$ in the Lagrangian density can be written as

$$
\phi=\sum_{i}\left(\frac{\dot{\alpha}_{i}}{2 \alpha_{i}}\left(r_{i}-d_{i}\right)^{2}+\dot{d}_{i}\left(r_{i}-d_{i}\right)\right),
$$

where an extra purely time-dependent additive contribution was ignored.

In addition, the Poisson equation admits an approximate solution given by

$$
V_{c}=-\sqrt{\frac{\pi}{2}} \frac{Q A}{\left(\alpha_{x} \alpha_{y} \alpha_{z}\right)^{1 / 3}} \frac{\operatorname{Erf}(\rho / \sqrt{2})}{\rho},
$$

where $\operatorname{Erf}(s)=\frac{2}{\sqrt{\pi}} \int_{0}^{s} \mathrm{~d} s^{\prime} \mathrm{e}^{-s^{\prime 2}}$ denotes the error function of a generic argument $s$. It is difficult to access the accuracy of the model approximation (11) in general. We consider just the case where $\alpha_{x}=\alpha_{y}=\alpha_{\perp}$ and define

$$
\bar{x}=\frac{x-d_{x}}{\alpha_{\perp}}, \quad \bar{y}=\frac{y-d_{y}}{\alpha_{\perp}}, \quad \bar{z}=\frac{z-d_{z}}{\alpha_{z}},
$$

together with $\bar{r}=\sqrt{\bar{x}^{2}+\bar{y}^{2}}$. The transformed Poisson equation assuming cylindrical symmetry is

$$
\begin{gathered}
\left(\frac{1}{\bar{r}} \frac{\partial}{\partial \bar{r}}\left(\bar{r} \frac{\partial}{\partial \bar{r}}\right)+\frac{\partial^{2}}{\partial \bar{z}^{2}}\right) V_{C}+\left(\frac{\alpha_{\perp}^{2}}{\alpha_{z}^{2}}-1\right) \frac{\partial^{2} V_{C}}{\partial \bar{z}^{2}}= \\
\frac{Q A}{\alpha_{z}} \exp \left(-\left(\bar{r}^{2}+\bar{z}^{2}\right) / 2\right) .
\end{gathered}
$$

It is apparent that the proposal (11) is an exact solution when $\alpha_{\perp}=\alpha_{z}$. In this case, the last term on the left hand side of (13) vanishes. The Ansatz is therefore more accurate provided $\alpha_{\perp} \simeq \alpha_{z}$. The same self-consistent potential form was adopted in the analysis of high-harmonic generation in a quantum electron gas trapped in a nonparabolic and anisotropic well [44].

In order to derive the dynamical behavior of the new coordinates, the Lagrangian is computed. After the substitution of (7), (10), (11) into (5) and (6), the following is obtained,

$$
\begin{gathered}
L\left(d_{i}, \dot{d}_{i}, \alpha_{i}, \dot{\alpha}_{i}\right) \equiv-\frac{1}{m N} \int \mathrm{d}^{3} r \mathcal{L}= \\
\mathrm{e}^{\nu t}\left[\sum_{i} \frac{1}{2}\left(\left(\dot{d}_{i}{ }^{2}+\dot{\alpha}_{i}{ }^{2}\right)-\nu \alpha_{i} \dot{\alpha}_{i}\right)-U_{d}-U_{\alpha}\right],
\end{gathered}
$$

where

$$
U_{d}=\sum_{i} \frac{\omega_{i}^{2}}{2} d_{i}^{2}
$$

and

$$
U_{\alpha}=\sum_{i}\left(\frac{\omega_{i}^{2}}{2} \alpha_{i}^{2}+\frac{b\left(\prod_{j} \alpha_{j}\right)^{\frac{1}{3}}}{8 \alpha_{i}^{2}}\right)+\frac{3 a}{2} \frac{1}{\left(\prod_{i} \alpha_{i}\right)^{\frac{2}{3}}}
$$


which are, respectively, the pseudo-potentials corresponding to the dipole and oscillating width modes, where the constants $a=3 \sqrt{3} k_{\mathrm{B}} T \alpha_{0}^{2} /(10 \sqrt{5} \pi m)$ and $b=N Q /\left(\pi^{3 / 2} m\right)$ are introduced and assuming that $\alpha_{i 0}=\alpha_{0}$. Also, in (16), the term $\sim \omega_{i}^{2}$ is related to the harmonic confinement, the term $\sim b$ corresponds to the self-consistent potential and the term $\sim a$ is due to the adiabatic pressure.

Once the Lagrangian is obtained, one can apply the Euler-Lagrange equations for each variational parameter, thus deriving the equations of motion. The dynamics of the center of mass is given by

$$
\ddot{d}_{i}+\nu \dot{d}_{i}+\omega_{i}^{2} d_{i}=0
$$

which, as can directly be seen, is decoupled to the width equations showing damped oscillations around the origin (damped Kohn oscillations). Furthermore, this motion is linear and independent of the number of atoms.

The equations of motion for the oscillating widths are as follows

$$
\begin{aligned}
& \ddot{\alpha}_{x}+\nu \dot{\alpha}_{x}+\left(\omega_{x}^{2}-\frac{\nu^{2}}{2}\right) \alpha_{x}=\frac{a}{\alpha_{x}^{5 / 3}\left(\alpha_{y} \alpha_{z}\right)^{2 / 3}}+\frac{b}{24}\left(\frac{5 \alpha_{y}^{1 / 3} \alpha_{z}^{1 / 3}}{\alpha_{x}^{8 / 3}}-\frac{\alpha_{y}^{1 / 3}}{\alpha_{x}^{2 / 3} \alpha_{z}^{5 / 3}}-\frac{\alpha_{z}^{1 / 3}}{\alpha_{x}^{2 / 3} \alpha_{y}^{5 / 3}}\right) \\
& \ddot{\alpha}_{y}+\nu \dot{\alpha}_{y}+\left(\omega_{y}^{2}-\frac{\nu^{2}}{2}\right) \alpha_{y}=\frac{a}{\alpha_{y}^{5 / 3}\left(\alpha_{x} \alpha_{z}\right)^{2 / 3}}+\frac{b}{24}\left(\frac{5 \alpha_{x}^{1 / 3} \alpha_{z}^{1 / 3}}{\alpha_{y}^{8 / 3}}-\frac{\alpha_{x}^{1 / 3}}{\alpha_{y}^{2 / 3} \alpha_{z}^{5 / 3}}-\frac{\alpha_{z}^{1 / 3}}{\alpha_{x}^{5 / 3} \alpha_{y}^{2 / 3}}\right),
\end{aligned}
$$

and

$$
\ddot{\alpha}_{z}+\nu \dot{\alpha}_{z}+\left(\omega_{z}^{2}-\frac{\nu^{2}}{2}\right) \alpha_{z}=\frac{a}{\alpha_{z}^{5 / 3}\left(\alpha_{x} \alpha_{y}\right)^{2 / 3}}+\frac{b}{24}\left(\frac{5 \alpha_{x}^{1 / 3} \alpha_{y}^{1 / 3}}{\alpha_{z}^{8 / 3}}-\frac{\alpha_{x}^{1 / 3}}{\alpha_{y}^{5 / 3} \alpha_{z}^{2 / 3}}-\frac{\alpha_{y}^{1 / 3}}{\alpha_{x}^{5 / 3} \alpha_{z}^{2 / 3}}\right) .
$$

The above set of equations apply for a confined atomic gas under arbitrary external harmonic confinement. In the following section, the applications of this method for MOTs will be pursued in more detail.

\section{The MOT case}

Confinement in MOTs has axially symmetric traps, $\omega_{x}=\omega_{y}=\omega_{\perp}$, so that it is allowed to take $\alpha_{x}=\alpha_{y}=\alpha_{\perp}$. In this way, the equations of motion become

$$
\begin{gathered}
\ddot{\alpha}_{\perp}+\nu \dot{\alpha}_{\perp}+\left(\omega_{\perp}^{2}-\frac{\nu^{2}}{2}\right) \alpha_{\perp}=\frac{a}{\alpha_{\perp}^{7 / 3} \alpha_{z}^{2 / 3}} \\
+\frac{b}{24}\left(\frac{4 \alpha_{z}^{1 / 3}}{\alpha_{\perp}^{7 / 3}}-\frac{1}{\alpha_{\perp}^{1 / 3} \alpha_{z}^{5 / 3}}\right)
\end{gathered}
$$

and

$$
\begin{gathered}
\ddot{\alpha}_{z}+\nu \dot{\alpha}_{z}+\left(\omega_{z}^{2}-\frac{\nu^{2}}{2}\right) \alpha_{z}=\frac{a}{\alpha_{\perp}^{4 / 3} \alpha_{z}^{5 / 3}} \\
+\frac{b}{24}\left(\frac{5 \alpha_{\perp}^{2 / 3}}{\alpha_{z}^{8 / 3}}-\frac{2}{\alpha_{\perp}^{4 / 3} \alpha_{z}^{2 / 3}}\right)
\end{gathered}
$$

or

$$
\ddot{\alpha}_{\perp}+\nu \dot{\alpha}_{\perp}=-\frac{1}{2} \frac{\partial U}{\partial \alpha_{\perp}}, \quad \ddot{\alpha}_{z}+\nu \dot{\alpha}_{z}=-\frac{\partial U}{\partial \alpha_{z}}
$$

where $U=U\left(\alpha_{\perp}, \alpha_{z}\right)$ is the pseudo-potential defined by

$$
\begin{gathered}
U\left(\alpha_{\perp}, \alpha_{z}\right)=\left(\omega_{\perp}^{2}-\frac{\nu^{2}}{2}\right) \alpha_{\perp}^{2}+\frac{1}{2}\left(\omega_{z}^{2}-\frac{\nu^{2}}{2}\right) \alpha_{z}^{2} \\
+\frac{3 a}{2 \alpha_{\perp}^{4 / 3} \alpha_{z}^{2 / 3}}+\frac{b}{8}\left(\frac{2 \alpha_{z}^{1 / 3}}{\alpha_{\perp}^{4 / 3}}+\frac{\alpha_{\perp}^{2 / 3}}{\alpha_{z}^{5 / 3}}\right),
\end{gathered}
$$

present in the associated Lagrangian function $\exp (\nu t)\left(\frac{1}{2}\left(2 \dot{\alpha}_{\perp}^{2}+\dot{\alpha}_{z}^{2}\right)-U\right)$.
From (21) and (22), the oscillating widths are described by the coupled nonlinear damped oscillator equations. In the undamped case $\nu=0$ and in the case with equal frequencies, $\omega_{\perp}=\omega_{z}$ corresponds to a Hamiltonian Ermakov system [52]. The nonlinearity comes from the repulsive interactions due to the pressure and self-consistent interaction (collective force) terms. The isotropic situation, when $\omega_{\perp}=\omega_{z}$ and $\alpha_{\perp}=\alpha_{z}$, corresponds to the radially symmetric case considered in [24], which uses a non-variational approach.

From the shape of the pseudo-potential (Fig. 1), one has that $\alpha_{\perp}$ and $\alpha_{z}$ will always execute damped oscillations around the unique minimum point $\left(\alpha_{\perp \text { eq }}, \alpha_{z \text { eq }}\right)$, since $a>0, b>0$. Looking for periodic oscillations restrict ourselves to $\omega_{\perp}>\nu / \sqrt{2}$ (which also implies $\omega_{z}>\nu / \sqrt{2}$ ), which are safely satisfied by MOT parameters.

One can solve (21) and (22) numerically for typical MOT parameters $[2,4,23,50,51]$. These are, namely, $T=0.3 \mathrm{mK}, \quad n_{0}=10^{16} \mathrm{~m}^{-3}$,

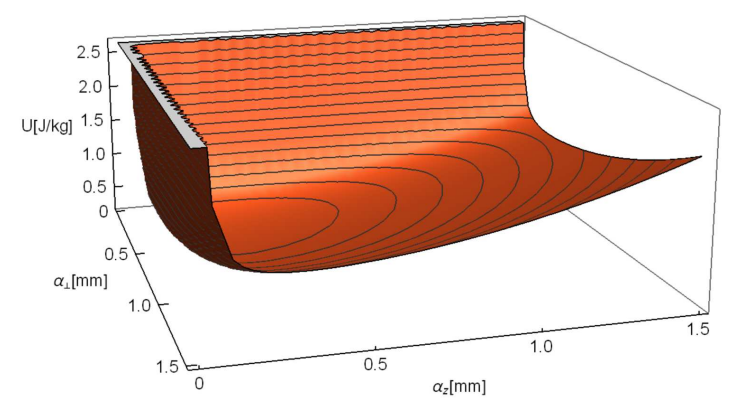

Fig. 1. Pseudo-potential from (24). Parameters are indicated in the text. 


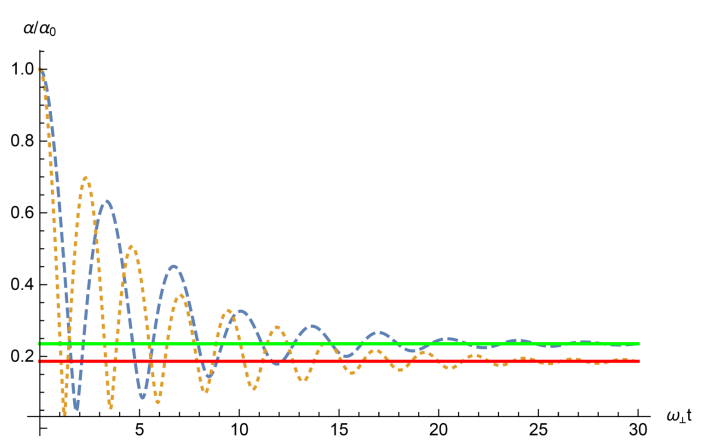

Fig. 2. Numerical solution of (21) and (22) normalized to $\alpha_{0}$. Parameters are indicated in the text. Dashed curve: $\alpha_{\perp}$; dotted curve: $\alpha_{z}$; full curves: equilibrium solutions $\alpha_{\perp \text { eq }} / \alpha_{0}=0.24$ and $\alpha_{z \text { eq }} / \alpha_{0}=0.19$. Initial conditions: $\alpha_{\perp 0}=\alpha_{z 0}=$ $\alpha_{0}$ and $\dot{\alpha}_{\perp 0}=\dot{\alpha}_{z 0}=0$.

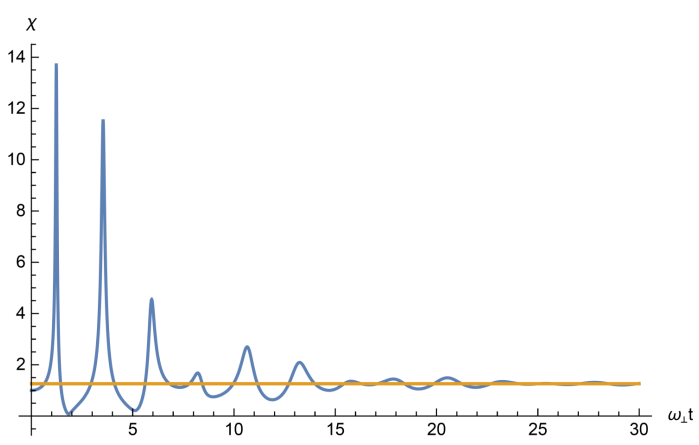

Fig. 3. Numerical simulation results for the anisotropy parameter $\chi$ with (25). Dashed curve: numerical simulation for $\chi$. The horizontal curve shows the equilibrium state $\chi_{\text {eq }}=$ $\alpha_{\perp \text { eq }} / \alpha_{z \text { eq }}=1.26$, using the same parameters as in Fig. 2.

$m=1.41 \times 10^{-25} \mathrm{~kg}$ (rubidium), $Q \sim 10^{-36} \mathrm{~N} \mathrm{~m}^{2}$, $\omega_{\perp}=697 \mathrm{rad} / \mathrm{s}, \nu=231 \mathrm{~s}^{-1}$, for $|\Delta|=2.5 \Gamma$, where $\Gamma=2 \pi \times 6 \mathrm{MHz}, k_{L} \sim 10^{7} \mathrm{~m}^{-1}, s_{\text {inc }}=0.1$, $|\nabla \boldsymbol{B}|=25 \mathrm{G} / \mathrm{cm}$, and $\alpha_{0}=1.5 \mathrm{~mm}$. The resulting damped nonlinear oscillations are shown in Fig. 2.

It is useful to consider a dimensionless function measuring the anisotropy,

$$
\chi=\frac{\alpha_{\perp}}{\alpha_{z}} .
$$

This is shown in Fig. 3 where the same parameters are used as in Fig. 2. It can be seen that anisotropy can be quite strong, contrary to the spherical symmetry assumption.

\section{Linear stability analysis}

For the linear stability analysis, restricted to the MOT case, rewrite (21) and (22) as

$$
\begin{aligned}
& \dot{\alpha}_{\perp}=\beta=f_{1}, \\
& \dot{\alpha}_{z}=\gamma=f_{2}, \\
& \dot{\beta}=-\nu \beta-\left(\omega_{\perp}^{2}-\frac{\nu^{2}}{2}\right) \alpha_{\perp}+\frac{a}{\alpha_{\perp}^{7 / 3} \alpha_{z}^{2 / 3}} \\
& \quad+\frac{b}{24}\left(\frac{4 \alpha_{\perp}^{1 / 3}}{\alpha_{z}^{7 / 3}}-\frac{1}{\alpha_{\perp}^{1 / 3} \alpha_{z}^{5 / 3}}\right)=f_{3}, \\
& \dot{\gamma}=-\nu \gamma-\left(\omega_{z}^{2}-\frac{\nu^{2}}{2}\right) \alpha_{z}+\frac{a}{\alpha_{\perp}^{4 / 3} \alpha_{z}^{5 / 3}} \\
& \quad+\frac{b}{24}\left(\frac{5 \sigma_{\perp}^{2 / 3}}{\sigma_{z}^{8 / 3}}-\frac{2}{\sigma_{\perp}^{4 / 3} \sigma_{z}^{2 / 3}}\right)=f_{4},
\end{aligned}
$$

or simply $\dot{\xi}_{m}=f_{m}$, for $m=1,2,3,4$, where $\xi_{m}=$ $\left(\alpha_{\perp}, \alpha_{z}, \beta, \gamma\right)$.

The stationary solutions corresponding to critical points of the pseudo-potential (24) are obtained by setting $\dot{\xi}_{m}=0$. These solutions satisfy the following equations,

$$
\begin{aligned}
& \left(\omega_{\perp}^{2}-\frac{\nu^{2}}{2}\right) \alpha_{\perp \text { eq }}-\frac{a}{\alpha_{\perp \text { eq }}^{7 / 3} \alpha_{z \text { eq }}^{2 / 3}} \\
& -\frac{b}{24}\left(\frac{4 \alpha_{z \text { eq }}^{1 / 3}}{\alpha_{\perp \text { eq }}^{7 / 3}}-\frac{1}{\alpha_{\perp \text { eq }}^{1 / 3} \alpha_{z \text { eq }}^{5 / 3}}\right)=0, \\
& \left(\omega_{z}^{2}-\frac{\nu^{2}}{2}\right) \alpha_{z \text { eq }}-\frac{a}{\alpha_{\perp \text { eq }}^{4 / 3} \alpha_{z \text { eq }}^{5 / 3}} \\
& -\frac{b}{24}\left(\frac{5 \alpha_{\perp \text { eq }}^{2 / 3}}{\alpha_{z \text { eq }}^{8 / 3}}-\frac{2}{\alpha_{\perp \text { eq }}^{4 / 3} \alpha_{z \text { eq }}^{2 / 3}}\right)=0,
\end{aligned}
$$

which can be numerically solved using $\beta_{\text {eq }}=0$, $\gamma_{\text {eq }}=0$.

Following the standard procedure, setting

$$
\xi_{m}=\xi_{m \text { eq }}+\delta \xi_{m} \exp (\mathrm{i} \Omega t)
$$

and linearizing around the equilibria, one derives

$$
\operatorname{det}(\text { i } \Omega \boldsymbol{I}-\boldsymbol{J})=0 \text {, }
$$

where $\boldsymbol{I}$ is the $4 \times 4$ identity matrix and $\boldsymbol{J}$ is the Jacobian matrix whose $(m, n)$ th entry is $J_{m n}=\left(\partial f_{m} / \partial \xi_{n}\right)_{\text {eq }}$ evaluated at equilibrium for $m, n=1,2,3,4$. In terms of the pseudo-potential in (24), the characteristic frequency $\Omega$ problem becomes

$\operatorname{det}\left(\begin{array}{cccc}\mathrm{i} \Omega & 0 & -1 & 0 \\ 0 & \mathrm{i} \Omega & 0 & -1 \\ \frac{1}{2}\left(\frac{\partial^{2} U}{\partial \alpha_{\perp}^{2}}\right)_{\mathrm{eq}} & \frac{1}{2}\left(\frac{\partial^{2} U}{\partial \alpha_{\perp} \partial \alpha_{z}}\right)_{\mathrm{eq}} & \mathrm{i} \Omega+\nu & 0 \\ \left(\frac{\partial^{2} U}{\partial \alpha_{\perp} \partial \alpha_{z}}\right)_{\text {eq }} & \left(\frac{\partial^{2} U}{\partial \alpha_{z}^{2}}\right)_{\text {eq }} & 0 & \mathrm{i} \Omega+\nu\end{array}\right)=0$ 
which can be managed to

$$
\begin{aligned}
& {\left[\left(\Omega-\frac{\mathrm{i} \nu}{2}\right)^{2}+\frac{\nu^{2}}{4}\right]^{2}-\left[\frac{1}{2} \frac{\partial^{2} U}{\partial \alpha_{\perp}^{2}}+\frac{\partial^{2} U}{\partial \alpha_{z}^{2}}\right]_{\mathrm{eq}}\left[\left(\Omega-\frac{\mathrm{i} \nu}{2}\right)^{2}+\frac{\nu^{2}}{4}\right]} \\
& \quad+\frac{1}{2}\left[\left(\frac{\partial^{2} U}{\partial \alpha_{\perp}^{2}}\right)_{\mathrm{eq}}\left(\frac{\partial^{2} U}{\partial \alpha_{z}^{2}}\right)_{\mathrm{eq}}-\left(\frac{\partial^{2} U}{\partial \alpha_{\perp} \partial \alpha_{z}}\right)_{\mathrm{eq}}^{2}\right]=0 .
\end{aligned}
$$

The quartic equation (35) can be immediately solved, yielding

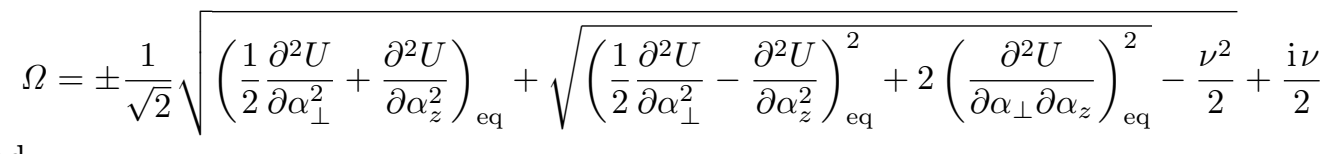

and

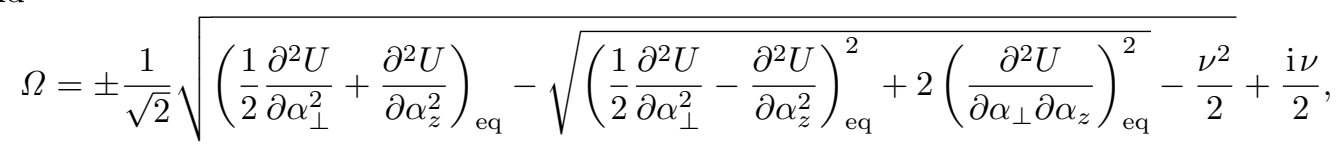

which for MOT parameters always corresponds to an asymptotically stable fixed point $(\operatorname{Re}(\Omega)>0$, $\operatorname{Im}(\Omega)>0)$. If nonlinearity and damping were negligible, one would find the solutions $\Omega^{2}=\omega_{\perp}^{2}$ and $\Omega^{2}=\omega_{z}^{2}$, as expected.

The asymptotically stable motion is expected from the shape of the pseudo-potential $U$, shown in Fig. 1. Using (36) and (37) within the parameters in Figs. 1-3, one finds $\operatorname{Im}(\Omega)=1.2 \times 10^{2} \mathrm{~s}^{-1}$ and $\operatorname{Re}(\Omega)= \pm 1.7 \times$ $10^{3} \mathrm{rad} / \mathrm{s}$ or $\operatorname{Re}(\Omega)= \pm 1.2 \times 10^{3} \mathrm{rad} / \mathrm{s}$.

\section{Conclusion}

In this work, atomic clouds confined in MOTs have been studied. The main result is the linear and nonlinear analysis of a trapped atomic gas in an anisotropic configuration. This is unlike most works in the literature, which are restricted to the spherically symmetric case that cannot be really adapted to MOTs. Our treatment was restricted to quadrupole fields created by a pair of anti-Helmholtz coils, which produced an azimuthal symmetric harmonic force. Moreover, the use of a variational approach for a dissipative system is a further distinctive feature of the present study. The basic equations for the axially symmetric threedimensional variational description have been derived, when thermal and multiple-scattering effects are both relevant. For this purpose, the starting point was a hydrodynamic set of equations reinterpreted in terms of the minimization of an action functional, adopting a Gaussian Ansatz. The stability analysis was performed obtaining the normal modes by solving the corresponding eigenvalue problem. Moreover, the results were applied to typical experiments and observed damped nonlinear coupled oscillations. In addition, the present approach can be directly adapted to non-neutral confined plasmas, where the damping mechanism can be traced back to collisions with neutrals.

\section{Acknowledgments}

L.G.F.S. and F.H. acknowledge the support of the Conselho Nacional de Desenvolvimento Científico e Tecnológico (CNPq). This study was financed in part by the Coordenação de Aperfeiçoamento de Pessoal de Nível Superior-Brasil (CAPES) Finance Code 001.

\section{References}

[1] M.H. Anderson, J.R. Ensher, M.R. Matthews, C.E. Wieman, E.A. Cornell, Science 269, 198 (1995).

[2] G. Labeyrie, F. Michaud, R. Kaiser, Phys. Rev. Lett. 96, 023003 (2006).

[3] A. di Stefano, M. Fauquembergue, P. Verkerk, D. Hennequin, Phys. Rev. A 67, 033404 (2003).

[4] C.G. Townsend, N.H. Edwards, C.J. Cooper, K.P. Zetie, C.J. Foot, A.M. Steane, P. Szriftgiser, H. Perrin, J. Dalibard, Phys. Rev. A 52, 1423 (1995).

[5] T. Pohl, G. Labeyrie, R. Kaiser, Phys. Rev. A 74, 023409 (2006).

[6] L. Guidoni, P. Verkerk, J. Opt. B: Quantum Semiclassical Opt. 1, R23 (1999).

[7] I. Bloch, Nat. Phys. 1, 23 (2005).

[8] S. Stellmer, B. Pasquiou, R. Grimm, F. Schreck, Phys. Rev. Lett. 110, 263003 (2013).

[9] R. Ayllon, H. Terças, J.T. Mendonça, Phys. Plasmas 26, 033501 (2019).

[10] B.B. Zelener, I.D. Arshinova, A.A. Bobrov, E.V. Vilshanskaya, S.A. Saakyan, V.A. Sautenkov, B.V. Zelener, V.E. Fortov, JETP Letters 108, 820 (2018).

[11] K. Gibble, S. Chu, Phys. Rev. Lett. 70, 1771 (1993). 
[12] G. Santarelli, Ph. Laurent, P. Lemonde, A. Clairon, A. G. Mann, S. Chang, A.N. Luiten, C. Salomon, Phys. Rev. Lett. 82, 4619 (1999).

[13] G. Labeyrie, E. Tesio, P.M. Gomes, G.-L. Oppo, W.J. Firth, G.R.M. Robb, A.S. Arnold, R. Kaiser, T. Ackemann, Nat. Photon. 8, 321 (2014).

[14] G. Manfredi, P.-A. Hervieux, Phys. Rev. Lett. 109, 255005 (2012).

[15] F. Haas, AIP Conf. Proc. 1421, 100 (2012).

[16] J.P. Cox, Theory of Stellar Pulsation, Princeton University Press, Princeton 1980.

[17] H. Terças, R. Kaiser, J.T. Mendonça, J. Loureiro, Phys. Rev. A 78, 013408 (2008).

[18] H. Terças, J.T. Mendonça, Phys. Rev. A 88, 023412 (2013).

[19] H. Terças, J.T. Mendonça, R. Kaiser, Europhys. Lett. 89, 53001 (2010).

[20] L. Pruvost, I. Serre, H.T. Duong, J. Jortner, Phys. Rev. A 61, 053408 (2000).

[21] R.S. de Oliveira, E.P. Raposo, S.S. Vianna, Phys. Rev. A 70, 023402 (2004).

[22] A. Fioretti, A.F. Molisch, J.H. Müller, P. Verkerk, M. Allegrini, Opt. Commun. 149, 415 (1998).

[23] A.S. Arnold, P.J. Manson, J. Opt. Soc. Am. B 17, 497 (2000).

[24] L.G.F. Soares, F. Haas, Phys. Scr. 94 , 125214 (2019).

[25] M. Gajda, J. Mostowski, Phys. Rev. A 49, 4864 (1994).

[26] A.M. Steane, M. Chowdhury, C.J. Foot, J. Opt. Soc. Am. B 9, 2142 (1992).

[27] P. Kohns, P. Buch, W. Süptitz, C. Csambal, W. Ertmer, Europhys. Lett. 22, 517 (1993).

[28] G.L. Gattobigio, T. Pohl, G. Labeyrie, R. Kaiser, Phys. Scr. 81, 025301 (2010).

[29] D.W. Sesko, T.G. Walker, C.E. Wieman, J. Opt. Soc. Am. B 8, 946 (1991).

[30] C.D. Wallace, T.P. Dinneen, K.Y.N. Tan, A. Kumarakrishnan, P.L. Gould, J. Javanainen, J. Opt. Soc. Am. B 11, 703 (1994).

[31] J.H. Müller, D. Ciampini, O. Morsch, G. Smirne, M. Fazzi, P. Verkerk, F. Fuso, E. Arimondo, J. Phys. B 33, 4095 (2000).
[32] J.L. Martin, C.R. McKenzie, N.R. Thomas, J.C. Sharpe, D.M. Warrington, P.J. Manson, W.J. Sandle, A.C. Wilson, J. Phys. B 32, 3065 (1999).

[33] J. Arlt, O. Maragò, E. Hodby, S.A. Hopkins, G. Hechenblaikner, S. Webster, C.J. Foot, J. Phys. B 33, 5861 (1999).

[34] P.O. Schmidt, S. Hensler, J. Werner, T. Binhammer, A. Görlitz, T. Pfau, J. Phys. B 5, S170 (2003).

[35] U. Ernst, A. Marte, F. Schreck, J. Schuster, G. Rempe, Europhys. Lett. 41, 1 (1998).

[36] J. Pérez-Ríos, A.S. Sanz, Am. J. Phys. 81, 836 (2013).

[37] S.K. Adhikari, Eur. Phys. J. D 42, 279 (2007).

[38] T.K. Ghosh, Eur. Phys. J. D 31, 101 (2004).

[39] F. Haas, B. Eliasson, J. Phys. B 51, 175302 (2018).

[40] L. Salasnich, Int. J. Mod. Phys. B 14, 1 (2000).

[41] L. Salasnich, J. Phys. A 42, 335205 (2009).

[42] V.M. Perez-Garcia, H. Michinel, J.I. Cirac, M. Lewenstein, P. Zoller, Phys. Rev. A 56, 1424 (1997).

[43] V.M. Perez-Garcia, H. Michinel, J.I. Cirac, M. Lewenstein, P. Zoller, Phys. Rev. Lett. 77, 5320 (1996).

[44] J. Hurst, K. Lévêque-Simon, P.A. Hervieux, G. Manfredi, F. Haas, Phys. Rev. A 93, 205402 (2016).

[45] G. Manfredi, P.A. Hervieux, F. Haas, New J. Phys. 14, 075012 (2012).

[46] P. Caldirola, Nuovo Cim. 18, 393 (1941).

[47] E. Kanai, Prog. Theor. Phys. 3, 440 (1948).

[48] T. Walker, D. Sesko, C. Wieman, Phys. Rev. Lett. 64, 408 (1990).

[49] M. Anwara, M. Faisal, M. Ahmed, Eur. Phys. J. D 67, 270 (2013).

[50] G.L. Gattobigio, F. Michaud, G. Labeyrie, T. Pohl, R. Kaiser, AIP Conf. Proc. 862, 211 (2006).

[51] T. Chaneliére, L. He, R. Kaiser, D. Wilkowski, Eur. Phys. J. D 46, 507 (2008).

[52] F. Haas, J. Goedert, J. Phys. A: Math. Gen. 29, 4083 (1996). 